
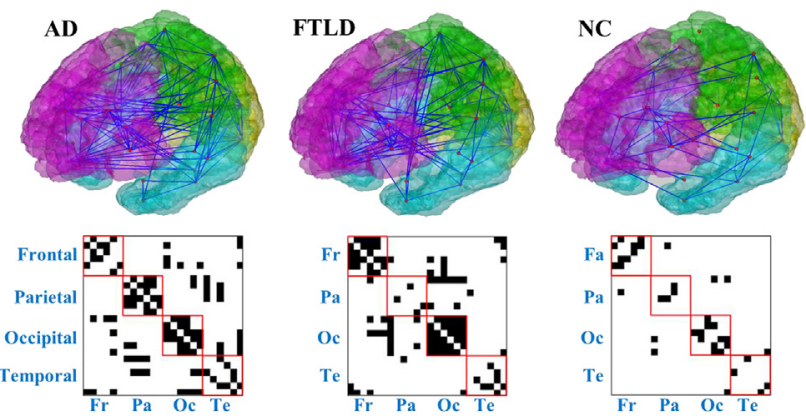

P4-298 IMPROVING THE POWER TO TRACK FIBRILLAR AMYLOID PET MEASUREMENTS AND EVALUATE AMYLOID-MODIFYING TREATMENTS USING A CEREBRAL WHITE MATTER REFERENCE REGION-OF-INTEREST

Kewei Chen ${ }^{1}$, Auttawut Roontiva ${ }^{2}$, Pradeep Thiyyagura ${ }^{3}$, Wendy Lee ${ }^{3}$, Xiaofen $\mathrm{Liu}^{3}$, Napatkamon Ayutyanont ${ }^{2}$, Hillary Protas ${ }^{4}$, Susan Landau ${ }^{5}$, Ji Luo $^{6}$, Robert Bauer, III, ${ }^{7}$, Cole Reschke ${ }^{3}$, Dan Bandy ${ }^{3}$, Robert Koeppe ${ }^{8}$, Adam S. Fleisher ${ }^{9}$, Richard J. Caselli ${ }^{10}$, William Jagust ${ }^{5}$,

Michael Walter Weiner ${ }^{11}$, Eric M. Reiman ${ }^{12},{ }^{1}$ Banner Alzheimer's Institute, Arizona State University, Arizona Alzheimer's Consortium, Phoenix, Arizona, United States, ${ }^{2}$ Banner Alzheimer's Institute, Arizona Alzheimer's Consortium, Phoenix, Arizona, United States, ${ }^{3}$ Banner Alzheimer's Institute, Arizona Alzheimer's Consortium, Phoenix, Arizona, United States; ${ }^{4}$ Banner Alzheimer's Institute, Arizona Alzheimer's Consortium, Phoenix, Arizona, United States; ${ }^{5}$ University of California, Berkeley, Berkeley, California, United States; ${ }^{6}$ Banner Alzheimer's Institute, Arizona Alzheimer's Consortium, Phoenix, Arizona, United States; ${ }^{7}$ Banner Alzheimers Institute, Arizona Alzheimer's Consortium, Phoenix, Arizona, United States; ${ }^{8}$ University of Michigan, Ann Arbor, Michigan, United States; ${ }^{9}$ Banner Alzheimer's Institute, Eli Lilly \& Co, Arizona Alzheimer's Consortium, Phoenix, Arizona, United States; ${ }^{10}$ Mayo Clinic, Arizona Alzheimer's Consortium, Scottsdale, Arizona, United States; ${ }^{11}$ Center for Imaging of Neurodegenerative Diseases, VA Medical Center and UCSF, San Francisco, California, United States; ${ }^{12}$ Banner Alzheimer's Institute, University of Arizona, Translational Genomics Research Institute, Phoenix, Arizona, United States. Contact e-mail: kewei.chen@bannerhealth.com

Background: We postulated that variability in longitudinal positron emission tomography (PET) measurements of fibrillar amyloid- $\beta(\mathrm{A} \beta)$ burden might be partly attributable to the combined effects of between-scan differences in head positioning and the use of inferior reference regions-of-interest (ROIs) in the computation of cerebral-to-reference ROI standard uptake value ratios (SUVRs). We used florbetapir PET images from the Alzheimer's Disease Neuroimaging Initiative (ADNI) to demonstrate improved power to track longitudinal fibrillar $\mathrm{A} \beta$ changes and evaluate $\mathrm{A} \beta$-modifying treatments using a cerebral white matter (WM) ROI. Methods: Baseline and 24-month follow-up florbetapir PET scans from 31 probable Alzheimer's dementia (pAD) patients, 187 mild cognitive impairment (MCI) patients and 113 cognitively normal controls (NCs) were used to compare the power of automatically generated cerebellar, pontine, and WM (eroded corpus callosum/centrum semiovale) reference ROIs to track SUVR changes and evaluate $\mathrm{A} \beta$-modifying treatment effects. Data were analyzed in $\mathrm{A} \beta+$ and $\mathrm{A} \beta-\mathrm{pAD}, \mathrm{MCI}$, and $\mathrm{NC}$ and cognitively normal apolipoprotein $\mathrm{E} 4$ (APOE4) carrier and non-carrier sub-groups. Results: In contrast to the use of cerebellar or pontine reference ROIs, the WM reference ROI permitted us to consistently detect significant longitudinal SUVR increases in the $\mathrm{A} \beta+\mathrm{pAD}, \mathrm{MCI}$, and $\mathrm{NC}$ and normal APOE4 carrier sub-groups, to consistently detect significantly greater SUVR increases in these groups than in their respective $\mathrm{A} \beta$ - or non-carrier controls, and to detect an overall correlation between longitudinal SUVR increases and longitudinal MiniMental State Examination (MMSE) score declines. Using the WM reference ROI, we estimate the need for far fewer pAD, MCI, A $\beta+\mathrm{NC}$, and APOE4- carrying NC subjects to detect an amyloid-modifying treatment effect in a 12-month placebo-controlled trial. Conclusions: A WM reference ROI can help improve the power to track longitudinal fibrillar $\mathrm{A} \beta$ increases, relate them to longitudinal cognitive decline, and evaluate $\mathrm{A} \beta$-modifying treatments with improved statistical power.

\section{P4-299 FIRST PROVEN OBSERVATION OF A DE NOVO PSEN1 MUTATION FROM GERMANY}

Felix Mueller-Sarnowski ${ }^{1}$, Jörg Meißner ${ }^{2}$, Christoph Laub ${ }^{3}$, Elisabeth Wlasich ${ }^{4}$, Theresa Raiser ${ }^{5}$, Axel Rominger ${ }^{6}$, Saskia Biskup ${ }^{7}$, Adrian Danek ${ }^{4},{ }^{1}$ Deutsches Zentrum für Neurodegenerative Erkrankungen, München, Germany; ${ }^{2}$ Private Practice, Sonneberg, Germany; ${ }^{3}$ Klinikum der Univeristät München, München, Germany; ${ }^{4}$ Klinikum der Universität München, München, Germany; ${ }^{5}$ Deutsches Zentrum für Neurodegenerative Erkrankungen, Munich, Germany; ${ }^{6}$ University of Munich LMU, Munich, Germany; ${ }^{7} \mathrm{CeGaT}$, Tübingen, Germany. Contact e-mail: felix. muellersarnowski@med.lmu.de

Background: A 49 year old male had developed cognitive impairment from age 44. According to ICD-10, DSMIV-TR and NINCDS-ADRDA AlzheimerÂ's dementia was probable from his clinical findings even though neuroimaging with MRI and FDG-PET had revealed no abnormalities. Total Tau and beta-amyloid in CSF were within normal range and only elevated phospho-Tau suggested Alzheimer pathology. Methods: Amyloid-PET using florbetaben, however, showed pronounced basal ganglia accumulation, which suggested a genetic correlate despite of a negative family history. There was no clinical correlate of the basal ganglia imaging findings. Molecular analysis in the patient uncovered a PSEN1 mutation. Results: Both parents (70 and 67 years old) were found unaffected on examination and neither showed the PSEN1 mutation. Further analyses proved that our patient was their true descendant. Thus, his L392V mutation has clearly arisen d e novo. Although their existence is not surprising, these mutations have yet rarely been observed in AlzheimerÂ's disease (Dumanchin et al. J Med Genet 1998 35(8):672-73) and our case report is the first one from Germany. Conclusions: In summary, genetic AlzheimerÂ's disease must be considered in all cases of early onset dementia and in particular if there is supporting evidence from the specific pattern of basal ganglia accumulation on amyloid imaging.

\section{P4-300 DEPRESSIVE SYMPTOMATOLOGY AS MEASURED BY THE PHQ9 IS LOW AMONG CLINIC PATIENTS RECEIVING A DIAGNOSIS OF ALZHEIMER DISEASE WITH OR WITHOUT AN AMYLOID PET SCAN}

Anil K. Nair ${ }^{1}$, Flavia Feijo ${ }^{2}$, Malini Nair ${ }^{3}$, Dhivya Meganathan ${ }^{4}$, Hayley Brooks ${ }^{3},{ }^{1}$ www.thealzcenter.org Alzheimer's Disease Center/Quincy Medical Center, Quincy, Massachusetts, United States;

${ }^{2}$ www.thealzcenterorg, Quincy, Massachusetts, United States;

${ }^{3}$ www.thealzcenter.org, Quincy, Massachusetts, United States;

${ }^{4}$ www.thealzcenter.org, Quincy, Massachusetts, United States.

Contacte-mail:nair.anil@gmail.com

Background: To identify if severity of depression symptoms as measured by PHQ9 questionnaire vary among memory clinic subjects receiving a diagnosis of Alzheimer dementia with and without amyloid PET scan results. Methods: A retrospective chart review identified all subjects attending the clinic from July 2011-July 2012. PHQ9 questionnaires were compiled and differences calculated among those receiving the diagnosis with and without an amyloid PET scan. Covariates such as age at memory complaint, gender, race, and education were also collected. Results: 298 patients attended the memory clinic from July 2011 to July 2012. 257(86.2\%) patients did not get and amyloid PET scan for diagnosis, 24 (8\%) subjects had positive amyloid PET scans and $17(5.7 \%)$ had negative amyloid PET scans. Mean age was 71.4 \pm 12.09 ; (71.48 \pm 8.08 among scan positives). Mean education was $12.76 \pm 2.98,13.13 \pm 1.77$ in the scan positive subjects. There were 174 (45.31\%) males; $11(45.8 \%)$ scan positive males. 242 (86.4\%) were Caucasian; 21 (100\%) scan positive Caucasians. 55 PHQ9s were available for review. Mean PHQ9 was 3.78 \pm 4.51 among all patients, 3.64 \pm 3.27 among scan positives and $4 \pm 4.87$ among scan negative subjects. Univariate analysis 